\title{
Development of inferences over elementary-school grades: I. Recall and association of implicit words
}

\author{
MELVIN H. MARX \\ Florida Institute of Technology, Melbourne, Florida
}

\begin{abstract}
Students in the second through the sixth grades were read a series of very short (one-sentence) "stories" by teachers in regular classroom settings. In each sentence, there was one clearly implied word. The students were then asked to write, cued by the title of each "story," one word that they had heard and one word that they had not heard but had thought of (and presumably inferred). A reliable increment over grades occurred in the number of implied words that were reported as thought of, with a sharp increment from the second to the third grades; and there was also a more regular increment over grades in the number of implied words that were used as associates to cues in a subsequent test.
\end{abstract}

Inferences, especially when defined as the filling in of gaps in verbal materials, are generally recognized as essential processes in the comprehension of discourse (see, e.g., Singer, 1988). For that reason, perhaps, their generation and subsequent use have been studied within a psycholinguistic framework; recognition of study sentences was the dependent variable most often used in the early research (e.g., Bransford \& Franks, 1971; Johnson, Bransford, \& Solomon, 1973; Paris \& Carter, 1973). These studies demonstrated subjects' ability to identify sentences from cues that had been implied in them but not explicitly included; for example, the cue spoon readily leads to the correct identification of the sentence "The truck driver stirred the coffee in his cup."

With respect to development, Paris and Lindauer (1976) reported that first graders were able to identify more sentences from explicit cues (that is, from the cue spoon when it had actually been in the studied sentence) than from implicit cues, but fifth graders were able to identify about as many such sentences from implicit as from explicit cues. The indication is that inferences are more readily generated and/or used by the older students.

Surprisingly, this problem does not seem to have been the object of much research done with standard learningmemory manipulations and measurements. Corbett and Dosher (1978) did examine protocols for implicit words, but they had subjects recall entire sentences.

This report is the first of several in which experiments on various facets of the development of inferences over the elementary-school grades have been addressed. The

\footnotetext{
I thank Rita Taubman, Director, and the participating teachers of St. Andrews Episcopal School, Ft. Pierce, FL, for their help in the collection of data and Arthur Gutman for the analyses of variance. Address correspondence to Melvin H. Marx, Department of Psychology, Florida Institute of Technology, 150 West University Blvd., Melbourne, FL 32901.
}

experiment described here represents a first attempt to have elementary-school students recall, or otherwise report on, words that are implicit in study sentences and therefore readily inferable. The major purpose was to track the development of such presumed inferences over the elementary-school grades. A second, parallel purpose was to assess the tendency to use such readily inferable words as associates for cues subsequently presented.

An additional objective was to determine whether the order in which the two tests-recall of words that had been heard and recall of words that had not been heard but had been thought of-would influence the number of inferences recalled or used as associates. Interference of recall of explicit information on problem solving has recently been reported by Brainerd and Reyna (1991), especially early in development. The test-order variable was therefore manipulated to see whether a similar kind of interference occurs when explicit recall ("heard" words) precedes recall of implicit ("'thought of') words.

\section{METHOD}

\section{Subjects}

The subjects were 76 elementary-school students in Grades $2(n=20)$, $3(n=18), 4(n=15), 5(n=17)$, and $6(n=6)$. Because the number of sixth graders was small, they were combined with the fifth graders in the data analysis. The mean ages (and $S D$ s) were $8.20(.44), 9.34(.57)$, $10.19(.47)$, and $11.40(.65)$ years for Grades 2 through 5-6, respectively.

\section{Experimental Design}

After the students had listened to the 16 sentences, with instructions to remember them because a memory test was to be given, performance on the two dependent variables was measured. The recall of implicit words was regarded as a test of explicit memory, in spite of the content of that memory, because the test was in a declarative format. The elicitation of implicit words was regarded as a test of implicit memory because the students were simply instructed to find words that made familiar phrases. The independent variables consisted of the elementary-school grade and the order in which the first two (explicit-memory) tests were given ("heard" words or "thought of" words to be listed first). 


\section{Stimulus Materials}

The 16 one-sentence "stories" are listed in Table 1, along with the (randomly determined) order in which they were read and with their titles. The following columns contain the presumed inferential (implicit) word for each sentence, the percentage of "correct" responses (that is, actual occurrences of the implicit words in the recall test), and the cue words that were intended to elicit implicit words as associates. In the preparation of the latter list, none of the primary associates for the cue words from the Jenkins (1970) norms were used; rather, in an effort to guard against ceiling effects, only cues with which the implicit words were moderately associated were selected.

\section{Procedure}

The teachers were given detailed procedural instructions, and they then collected the data in their own intact classes. The students were told that they were to listen to a series of "short stories" and that they should try to remember them because they would be asked questions about them later. The first sentence ("The Delivery Man") was for practice: "On the night before Christmas a large figure with a flowing white beard and a red suit delivers boxes to homes with children." (This story is not included in Table 1.) After the sentences had all been read, with their numbered titles and with a 2-sec pause between sentences, answer sheets were passed out. The two types were alternated along rows, with instructions to work from left to right, either "heard" or "thought of " words coming first. The students were asked to fill in their birthdates and other information at the top of the page. Two columns were labeled WORDS I HEARD and WORDS I THOUGHT OF, with the numbers and titles of the sentences listed in a column on the left margin. The testing procedure was explained and the practice sentence repeated. The teacher enumerated some of the more obviously inferable words (e.g., "Santa Claus," "reindeer," "sleigh") and asked the students to write one word in each column for this first "story." They were then instructed to continue to use the same procecture for each of the remaining "stories," writing one important word in each space. Approximately $5 \mathrm{~min}$ were allowed for this task. The students were next instructed to turn over their answer sheets and look at the list of 17 word cues, each of which was followed by a blank space. They were told to write one word in the space after each cue, so as to make a familiar phrase (as, e.g., "Claus" after the practice-story cue "Santa"). Additional examples were given (e.g., the cue "home," which could be followed by "room," "town," "plate," and the like). The students were asked to work on the remaining 16 cues in the same way and were allowed about $5 \mathrm{~min}$ to do so. Only procedural questions were answered; the teachers were not aware of the purpose of the experiment.

\section{RESULTS}

\section{Recall of Implicit Words}

There was a sharp increase in the percentage of students who recalled at least one of the implicit words (intended inferences) from the second to the third grades: 8 of $20(40 \%)$ did so in the second grade, compared with 17 of $18(94 \%)$ in the third grade. The difference in proportions was reliable $\left[\chi^{2}(1)=12.13, p<.01\right]$.

The mean number of implicit words recalled at each grade level is shown in Figure 1. The grade variable was a reliable main effect in an analysis of variance $[F(3,68)=$ $6.517, p<.001]$.

The test-order variable was not reliable $[F(1,68)=$ $2.477, p>.05]$. In any event, the means were opposite to the interference prediction ( 2.27 for the 37 subjects who recalled their "heard" words first-and so should have suffered from the interference on their subsequent recall of the presumed inferences-and 1.82 for the 39 subjects reporting the inferences first). There was no interaction between grade and test-order variables $(F<1.0)$.

Table 1 provides information on the degree of successful recall of implicit words in the various sentences. It can be seen that four of the words were complete duds, and another two very nearly so (only one recall each). The burden of differentiation in these data was obviously borne by the four most productive implicit words: bird ("feeder"), worm ("bait"), juice ("citrus"), and water ("oasis").

\section{Association of Implicit Words}

A small but steady increment over grades was found in the use of implicit words as associates. A lower proportion of students in Grades 2 and 3 (23 of 38, or $61 \%$ ) made at least one such association, compared with students in Grades 4 and 5-6 (33 of 38, or 82\%). The difference was reliable $\left[\chi^{2}(1)=6.79, p<.05\right]$.

Table 1

Titles and Study Sentences, Intended Implicit Words, Percentage Recalled, and Cue Words

\begin{tabular}{|c|c|c|c|}
\hline Titles: Study Sentences & $\begin{array}{l}\text { Implicit } \\
\text { Words }\end{array}$ & \% Recalled & $\begin{array}{l}\text { Cue } \\
\text { Words }\end{array}$ \\
\hline 2. The Hook: The boy put a wiggly bait on the hook. & worm & 28 & EARTH \\
\hline 3. The Fisherman: The fisherman sliced the large fish into equal sections. & knife & 7 & BREAD \\
\hline $\begin{array}{l}\text { 4. The Shopper: The shopper's pen ran out, just before she could sign the } \\
\text { check. }\end{array}$ & ink & 7 & DARK \\
\hline 5. The Worker: The tired worker sat down heavily, at the dinner table. & chair & 0 & HIGH \\
\hline 6. The Seeds: All the seeds in the feeder had been eaten & bird & 36 & BLUE \\
\hline $\begin{array}{l}\text { 7. The Gardener: The gardener washed and wiped her hands dry before starting } \\
\text { to cook supper. }\end{array}$ & towel & 0 & BATH \\
\hline 8. The Travelers: The thirsty travelers found an oasis in the desert. & water & 16 & CITY \\
\hline 9. The Guests: The housewife squeezed citrus for her unexpected guests. & juice & 18 & FRUIT \\
\hline $\begin{array}{l}\text { 10. The Lifeguard: The lifeguard heard the swimmer but couldn't see him in the } \\
\text { dim light. }\end{array}$ & shout & 0 & LOUD \\
\hline 11. The Sailor: The sailor grabbed the oars and rowed towards the boy. & boat & 9 & HOUSE \\
\hline 12. The Teenager: The teenager shampooed almost daily. & hair & 5 & LONG \\
\hline 13. The Mother: The mother poured hot chocolate for her sleepy child. & cup & 0 & BUTTER \\
\hline 14. The Jockey: The jockey threw on a saddle and rode off at a gallop. & horse & 26 & HUNGRY \\
\hline 15. The Assignment: The teacher took some chalk and wrote the assignment. & board & 1 & BLACK \\
\hline 16. The Coke: All the Coke spilled of & bottle & 1 & BABY \\
\hline 17. The Party: Each child was given a large serving, baked especially & cake & 9 & SHORT \\
\hline
\end{tabular}
birthday party, along with ice cream and soda pop. 


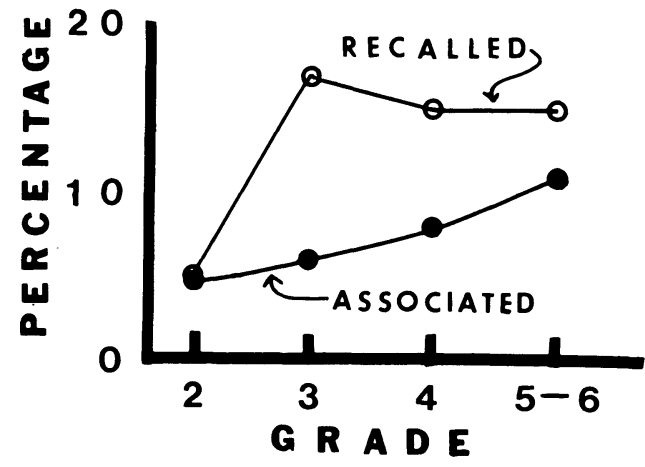

Figure 1. Mean percentage of implicit words that were recalled and associated as a function of elementary-school grade.

The mean number of implicit-word associations over grades is shown in Figure 1. The grade variable was a reliable main effect $[F(3,68)=5.225, p<.01]$. Neither test order nor its interaction with grade was reliable $(F<1.0)$.

There was considerable variability in the elicitation of implicit words by the cues. The most frequently elicited implicit words were bird (18 occurrences), cup (14), juice (13), and cake (11); only two of the implicit words were not used (ink and chair). The most successful cues were BLUE (17 implicit words elicited, 15 of which were the intended bird) and BUTTER (15 elicitations, 7 of which were the intended cup).

\section{Additional Analyses}

Two other results deserve mention. First, productmoment correlations were computed between the recall and association scores, separately for each grade group. Only slight, variable $r$ s were found: $.08, .20,-.12$, and -.05 for Grades 2 through 5-6, respectively.

Second, explicit-word recalls were scored, but this measure was too undemanding (only one "heard" word requested) to allow much differentiation among grades. Nevertheless, there was some improvement over grades, at least again from Grade 2: means were 11.8, 14.2, 15.0, and 15.1 for Grades 2, 3, 4, and 5-6, respectively. A chisquare analysis suggested the reliability of the difference between Grades 2 and 3 in the proportion of students whose scores exceeded the rounded mean (13) for all students: 8 of 20 second graders (40\%), compared with 14 of 18 third graders $(82 \%)$ [ $\left.\chi^{2}(1)=5.62, p<.05\right]$.

\section{DISCUSSION}

The present results suggest that both the recall (explicit-memory test) and associative use (implicit-memory test) of implicit words from studied sentences increase over the elementary-school grades. The evidence is clearest for the early grades, in accordance with previous results (e.g., Paris \& Lindauer, 1976) based on the use of sentence identification. Why older students are more effective at remembering and using implicit words is an interesting and important question-one among many that need to be attacked experimentally. Are older students simply better at making inferences? Or do they encode and/or retrieve inferences more effectively? Experimental techniques that more immediately probe for report or other evidence of the encoding of implicit words need to be developed.

Graf's recent (1990) suggestion of developmental invariance for implicit memory would seem to be contradicted by the reliable increment found in the association measure, here regarded as an implicit-memory test. However, this conclusion cannot be advanced very strongly, because there was no control for normal developmental improvement in this kind of test; and there was a multiplicity of associations between cues and implicit words. The use of weakly associated eliciting cues should have reduced any tendency to make these associations-rather, other more strongly associated words would be expected to increase with intellectual development. Nevertheless, the question can best be resolved by the pinpointing of associations between implicit words in study and their subsequent selection in an implicit-memory test, relative to comparable nonstudied words.

The failure of the two memory tests to show any tendency toward correlation suggests that the mechanisms underlying them may be substantially independent. Also, the failure of the test-order variable to reveal interference effects, on either the explicit or the implicit test, is consistent with that proposition. However, these are at best highly tentative conclusions; in particular, a more rigorous experimental test of possible interference effects than the simple manipulation that was used will be required for any firmer conclusions.

\section{REFERENCES}

Brainerd, C. J., \& Reyna, V. F. (1991). Memory independence and memory interference in cognitive development. Manuscript submitted for publication.

Bransford, J. D., \& Franks, J. J. (1971). The abstraction of linguistic ideas. Cognitive Psychology, 2, 331-350.

Corbett, A. T., \& Dosher, B. A. (1978). Instrument inferences in sentence encoding. Journal of Verbal Learning \& Verbal Behavior, 17, 479-492.

GraF, P. (1990). Life-span changes in implicit and explicit memory. Bulletin of the Psychonomic Society, 28, 353-358.

Jenkins, J. J. (1970). The 1952 Minnesota Word Association Norms. In L. Postman \& G. Keppel (Eds.), Norms of word association. New York: Academic Press.

Johnson, M. K., Bransford, J. D., \& Solomon, S. K. (1973). Memory for tacit implications of sentences. Journal of Experimental Psychology, 98, 203-205.

PARIS, S. G., \& CARTER, A. Y. (1973). Semantic and constructive aspects of sentence memory in children. Developmental Psychology, 9, 109-113.

Paris, S. G., \& Lindauer, B. K. (1976). The role of inference in children's comprehension and memory for sentences. Cognitive Psychology, 8, 217-227.

SINGER, M. (1988). Inferences in reading comprehension. In M. Daneman, G. MacKinnon, \& T. Waller (Eds.), Reading research: Advances in theory and practice (Vol. 6, pp. 177-219). New York: Academic Press. 